\title{
THE STRICT TOPOLOGY AND COMPACTNESS IN THE SPACE OF MEASURES. II
}

\author{
BY \\ JOHN B. CONWAY( $\left.{ }^{1}\right)$
}

The strict topology $\beta$ on $C(S)$, the bounded continuous complex valued functions on the locally compact Hausdorff space $S$, was first introduced by R. C. Buck [3], [4], [5]. It has also been studied by I. Glicksberg [10], J. Wells [20], and C. Todd [17]. This topology has been used in the study of various problems in spectral synthesis [11], spaces of bounded holomorphic functions [15], and multipliers of Banach algebras [18], [19]. This paper is a detailed account of results announced by the author in [6], [7] on the relationship of $C(S)_{\beta}$ with its dual $M(S)$, the bounded Radon measures on $S$. In particular, we are concerned with the question (posed by Buck) of whether or not $C(S)_{\beta}$ is a Mackey space and, consequently, with compactness criteria in $M(S)$.

The existence and description of the Mackey topology, the strongest topology yielding a given adjoint space, is known, and there are several properties (e.g., metrizable) which imply that a designated topology is the Mackey topology. However, the author knows of no example of a topological vector space with an intrinsically defined topology which is a Mackey space, except by virtue of some formally stronger property (e.g., metrizable, barrelled, bornological). This is not true for $C(S)_{\beta}$. In fact, we show that if $S$ is paracompact then every $\beta$-weak* countably compact subset of $M(S)$ is $\beta$-equicontinuous; consequently, $C(S)_{\beta}$ is a Mackey space (Theorem 2.6). Also, if $S$ is not compact then $C(S)_{\beta}$ is not barrelled, bornological, nor metrizable. It can also happen that $C(S)_{\beta}$ is not a Mackey space, as we show for the case when $S$ is the space of ordinal numbers less than the first uncountable ordinal.

In $\S 3$ we examine the subspace problem for $C(S)_{\beta}$. That is, if $C(S)_{\beta}$ is a Mackey space, which subspaces of $C(S)$ are Mackey spaces when furnished with the relative strict topology? We are able to solve this problem when $S$ is the space of positive integers. Also, we show that $H^{\infty}$, the bounded holomorphic functions on the open unit disk $D$, is not a Mackey space when endowed with the $\beta$ topology-even though $C(D)_{\beta}$ is. From these results we prove the existence of a closed subspace $N$ of $l^{1}$ such that there is no bounded projection of $l^{1}$ onto $N$. Finally, $\left(l^{\infty}, \beta\right)$ is a semireflexive Mackey space with a closed subspace which is not a Mackey space.

Received by the editors August 20, 1965 and, in revised form, July 13, 1966.

(1) These are some of the results from the author's doctoral dissertation written while he held a National Science Foundation Cooperative Fellowship at Louisiana State University. Partial support was also furnished by NSF Grant GP 1449. The author would like to thank Professor Heron S. Collins for his advice and especially his encouragement. 
1. Notation and preliminaries. We will let $C_{0}(S)$ denote the subspace of $C(S)$ consisting of all those functions which vanish at infinity, and $C_{\mathrm{c}}(S)$ those which vanish off some compact set. If $\phi$ is in $C(S)$ then $N(\phi)=\{s: \phi(s) \neq 0\}$, spt $(\phi)=$ the closure of $N(\phi)$, and $\|\phi\|_{\infty}=\sup \{|\phi(s)|: s \in S\}$. Finally, $V_{\phi}=\left\{f \in C(S):\|\phi f\|_{\infty} \leqq 1\right\}$.

The strict topology $\beta$ on $C(S)$ has as a neighborhood basis at the origin the collection of sets

$$
\left\{V_{\phi}: \phi \in C_{0}(S), \phi \geqq 0\right\} ;
$$

that is, it is defined by the seminorms $f \rightarrow\|\phi f\|_{\infty}$ on $C(S)$, where $\phi \in C_{0}(S)$. The compact open topology on $C(S)$ (denoted by c-op) has as a neighborhood basis at the origin the sets of the form $V_{\phi}$ where $\phi \in C_{\mathrm{c}}(S)$ and $\phi \geqq 0$. It is known that $C(S)_{\beta}$ is complete, $C_{\mathrm{c}}(S)$ is $\beta$-dense in $C(S)$, the norm bounded and $\beta$-bounded subsets of $C(S)$ are the same, the $\beta$ and c-op topologies agree on norm bounded subsets of $C(S)$, and $C(S)_{\beta}^{*}=M(S)$ where the correspondence is by integration (see [5] for the proofs of these and other basic facts about the strict topology).

A topological vector space $E$ is a Mackey space if and only if every weak* compact convex circled subset of $E^{*}$ is equicontinuous. We will say that $E$ is a strong Mackey space if and only if every weak* compact subset of $E^{*}$ is equicontinuous.

If $A$ is a subset of a topological space $X$ then $A$ is sequentially compact if and only if every sequence in $A$ has a subsequence which converges to some point in $X$; $A$ is countably compact if and only if every sequence in $A$ has a cluster point in $X$; $A$ is conditionally compact if and only if $A^{-}$(the closure of $A$ ) is compact. Our remaining terminology will be standard as is found in [8], [13], and [12].

2. The Mackey topology on $C(S)_{\beta}$ and the main theorem. In order to discover whether or not $C(S)_{\beta}$ is a Mackey space we must first characterize the $\beta$-equicontinuous subsets of $M(S)$. A set $H \subset M(S)$ is $\beta$-equicontinuous if and only if

$$
H^{0} \equiv\left\{f \in C(S):\left|\int f d \mu\right| \leqq 1 \text { for all } \mu \in H\right\}
$$

is a $\beta$-neighborhood of zero in $C(S)$. Since the sets $V_{\phi}$ form a neighborhood basis at zero, this is equivalent to saying that $V_{\phi} \subset H^{0}$ for some $\phi \in C_{0}(S)$; or, $H \subset V_{\phi}^{0} \subset M(S)$ where

$$
V_{\phi}^{0} \equiv\left\{\mu \in M(S):\left|\int f d \mu\right| \leqq 1 \text { for all } f \in V_{\phi}\right\}
$$

Hence we must characterize the sets $V_{\phi}^{0}$ where $\phi$ is in $C_{0}(S)$. This is done in the following theorem of I. Glicksberg [10]. For the outline of a proof based on functional analysis rather than measure theory the reader may refer to $[6$, Theorem 1].

THEOREM 2.1. If $\phi$ is in $C_{0}(S)$ then

$$
V_{\phi}^{0}=\{\mu \in M(S): \mu \text { vanishes off } N(\phi) \text { and }\|\mu / \phi\| \leqq 1\} \text {. }
$$

(Here, $\mu / \phi$ denotes the measure $\nu$ such that $\nu(A)=\int_{A}(1 / \phi) d \mu$.) 
This criterion for $\beta$-equicontinuity is, however, not sufficient for our purposes. An alternate characterization, which will be used in the proof of our main theorem, is provided by the following.

THEOREM 2.2. A set $H \subset M(S)$ is $\beta$-equicontinuous if and only if $H$ is uniformly bounded and for every $\varepsilon>0$ there is a compact set $K \subset S$ such that $|\mu|(S \backslash K) \leqq \varepsilon$ for all $\mu$ in $H$.

Proof. Suppose that $H$ is $\beta$-equicontinuous; then there is a nonnegative function $\phi$ in $C_{0}(S)$ such that $H \subset V_{\phi}^{0}$. It easily follows from Theorem 2.1 that $H$ is uniformly bounded by $\|\phi\|_{\infty}$. If $\varepsilon>0$ and we let $K=\{s: \phi(s) \geqq \varepsilon\}$ then $K$ is compact and, again applying Theorem 2.1, we have that

$$
\begin{aligned}
|\mu|(S \backslash K) & =\int_{S \mid K} \phi(1 / \phi) d|\mu| \\
& \leqq\|\mu / \phi\| \sup \{\phi(s): s \notin K\} \\
& \leqq \varepsilon .
\end{aligned}
$$

For the converse suppose that $\|\mu\| \leqq 1$ for all $\mu$ in $H$ and that the condition is satisfied. By induction we can obtain a sequence $\left\{K_{n}\right\}$ of compact subsets of $S$ such that $K_{n} \subset$ int $K_{n+1}$ (the interior of $\left.K_{n+1}\right)$ and $|\mu|\left(S \backslash K_{n}\right) \leqq\left(\frac{1}{4}\right)^{n}$ for all $\mu$ in $H$. For each integer $n \geqq 1$ let $\phi_{n} \in C_{\mathrm{c}}(S)$ such that $\phi_{n}\left(K_{n}\right)=1,0 \leqq \phi_{n} \leqq 1$, and $\phi_{n}(s)=0$ for $s \notin K_{n+1}$. Put

$$
\phi(s)=2 \sum_{n=1}^{\infty}\left(\frac{1}{2}\right)^{n} \phi_{n}(s), \quad s \in S .
$$

Then $\phi \in C_{0}(S)$ and $N(\phi)=\bigcup_{n=1}^{\infty} K_{n}$. We will now show that $H$ is contained in $V_{\phi}^{0}$ and is, therefore, $\beta$-equicontinuous. If $A \in \operatorname{Borel}(S)$ (the Borel subsets of $S$ ) and $A \cap N(\phi)=\square$ then $A \cap K_{n}=\square$ for all $n \geqq 1$; thus $|\mu|(A) \leqq\left(\frac{1}{4}\right)^{n}$ for all $n$ and so each $\mu$ in $H$ vanishes off $N(\phi)$. If $s \in K_{n} \backslash K_{n-1}, n \geqq 2$, then $\phi_{1}(s)=\cdots=\phi_{n-2}(s)=0$ and $\phi_{k}(s)=1$ for $k \geqq n$. Therefore

$$
\begin{aligned}
\phi(s) & =2 \sum_{k=n-1}^{\infty}\left(\frac{1}{2}\right)^{k} \phi_{k}(s) \\
& \geqq 2 \sum_{k=n}^{\infty}\left(\frac{1}{2}\right)^{k}=2\left(\frac{1}{2}\right)^{n-1} .
\end{aligned}
$$

If $\mu$ is in $H$ and $n \geqq 2$ then

and so

$$
\int_{K_{n} \mid K_{n-1}}(1 / \phi) d|\mu| \leqq\left(\frac{1}{2}\right) 2^{n-1}\left(\frac{1}{4}\right)^{n-1} \leqq\left(\frac{1}{2}\right)^{n}
$$

$$
\begin{aligned}
\int(1 / \phi) d|\mu| & =\int_{K_{1}}(1 / \phi) d|\mu|+\sum_{n=2}^{\infty} \int_{K_{n} \backslash K_{n-1}}(1 / \phi) d|\mu| \\
& \leqq\left(\frac{1}{2}\right)\|\mu\|+\sum_{n=2}^{\infty}\left(\frac{1}{2}\right)^{n} \leqq 1
\end{aligned}
$$

This completes the proof of the theorem. 
The plan for proving Theorem 2.6 will be to first prove it when $S$ is the space of positive integers and then reduce the general case to this one.

LEMMA 2.3. The strong topology on $M(S)=C(S)_{B}^{*}$ is exactly the norm topology. Hence $C_{0}(S)$ and $C(S)_{\beta}$ have the same second adjoint space. Also, $C(S)_{\beta}$ is semireflexive if and only if $S$ is discrete.

Proof. The strong topology on $M(S)=C(S)_{\beta}^{*}$ is, by definition, the topology of uniform convergence on $\beta$-bounded subsets of $C(S)$. But the $\beta$-bounded and norm bounded subsets of $C(S)$ are the same [5, p. 98], and so the strong topology on $M(S)$ is the norm topology.

If $S$ is discrete then it is immediate that $C(S)_{\beta}$ is semireflexive. For the converse let $s \in S$ and put $L(\mu)=\mu(\{s\})$ for all $\mu$ in $M(S)$. Then $L \in M(S)^{*}$ so that if $C(S)_{\beta}$ is semireflexive there is a function $f$ in $C(S)$ such that $L(\mu)=\int f d \mu$ for all $\mu \in M(S)$. It now follows that $f$ is the characteristic function of $\{s\}$, which, consequently, must be open. This completes the proof.

Note that if $S$ is the space of positive integers with the discrete topology then $C(S)=l^{\infty}, M(S)=l^{1}$, and $C_{0}(S)=c_{0}$. Also, from Theorem 2.2, a set $H \subset l^{1}$ is $\beta$-equicontinuous if and only if it is bounded and for every $\varepsilon>0$ there is an integer $N$ such that $\sum_{n=N+1}^{\infty}\left|a_{n}\right| \leqq \varepsilon$ for every $\alpha=\left\{a_{n}\right\}_{n=1}^{\infty}$ in $H$.

Before proceeding let us make a distinction between two weak star topologies on $M(S)$. We will denote by $\beta$-weak the weak star topology which $M(S)$ has as the dual of $C(S)_{\beta}$. This will distinguish it from the weak* topology which $M(S)$ has as the dual of the Banach space $C_{0}(S)$.

THEOREM 2.4. If $H \subset l^{1}$ then the following are equivalent:

(a) $H$ is weakly conditionally compact;

(b) $H$ is $\beta$-weak* conditionally compact;

(c) $H$ is norm conditionally compact;

(d) $H$ is $\beta$-equicontinuous.

Proof. It is clear that (a) and (b) are equivalent because $\left(l^{\infty}, \beta\right)$ is semireflexive; also it is trivial that (d) implies (b). To see that (a) implies (c) let $\left\{\xi_{n}\right\}$ be a sequence in $H$. Then there is a subsequence $\left\{\xi_{n_{k}}\right\}$ of $\left\{\xi_{n}\right\}$ which converges weakly to some element $\xi$ of $l^{1}[8$, p. 430]. But it is a well-known result of J. Schür (e.g., see p. 296 of [8]) that weak and norm sequential convergence are the same in $l^{1}$. Thus, $\left\{\xi_{n_{k}}\right\}$ converges to $\xi$ in norm and $H$ is norm conditionally compact.

If (c) holds and we wish to establish (d) then let $\varepsilon>0$ and choose $\xi_{1}, \ldots, \xi_{n}$ in $H$ such that

$$
H \subset \bigcup_{k=1}^{n}\left\{\xi:\left\|\xi-\xi_{k}\right\|<\varepsilon / 2\right\} .
$$

If for $k=1, \ldots, n$ we let $\xi_{k}=\left\{x_{i}^{(k)}\right\}_{i=1}^{\infty}$ then we may find an integer $N$ such that

$$
\sum_{i=N+1}^{\infty}\left|x_{i}^{(k)}\right|<\varepsilon / 2
$$


for $1 \leqq k \leqq n$. From here it easily follows that

$$
\sum_{i=N+1}^{\infty}\left|x_{i}\right|<\varepsilon
$$

for all $\xi=\left\{x_{i}\right\}_{i=1}^{\infty}$ in $H$. This completes the proof of the theorem.

COROLlaRY 2.5. The space $\left(l^{\infty}, \beta\right)$ is a strong Mackey space.

We are now in a position to prove the principal result of this paper.

THEOREM 2.6. If $S$ is paracompact and $H \subset M(S)$ is $\beta$-weak* countably compact then $H$ is $\beta$-equicontinuous. Consequently, $C(S)_{\beta}$ is a strong Mackey space whenever $S$ is paracompact.

Proof. We will prove the theorem for the case when $S$ is $\sigma$-compact (i.e., $S$ is the countable union of compact sets), and, afterwards, indicate the proof for the general case. Therefore, let $S=\bigcup_{n=1}^{\infty} D_{n}$ where each $D_{n}$ is compact and $D_{n} \subset$ int $D_{n+1}$. Suppose that $H$ is not $\beta$-equicontinuous. Since $H$ is $\beta$-weak* countably compact, it follows that $H$ is weak* bounded and hence uniformly bounded. Thus, Theorem 2.2 implies there is an $\varepsilon>0$ such that for every compact set $K \subset S,|\mu|(S \mid K)>\varepsilon$ for some $\mu$ in $H$. We can now prove the following

Claim 1. There exists a sequence of quadruples $\left\{\left(\mu_{n}, \phi_{n}, K_{n}, U_{n}\right)\right\}_{n=1}^{\infty}$ having the following properties:

(a) $\mu_{n} \in H, \phi_{n} \in C_{\mathrm{c}}(S), K_{n}$ is compact, $U_{n}$ is open in $S$ with $U_{n}^{-}$compact and $U_{n}^{-} \cap K_{n}=\square$;

(b) $D_{n} \cup K_{n} \cup U_{n}^{-} \subset$ int $K_{n+1}$;

(c) $\left|\mu_{n}\left(U_{n}\right)\right|>\varepsilon / 4$;

(d) $\left\|\phi_{n}\right\|_{\infty}=1$, spt $\left(\phi_{n}\right) \subset U_{n}$, and $\left|\mu_{n}\right|\left(U_{n}\right)<\left|\int \phi_{n} d \mu_{n}\right|+\varepsilon / 8$.

If this is established, we can then prove the following facts.

Claim 2. $S=\bigcup_{n=1}^{\infty}$ int $K_{n}$.

Claim 3. $F=\bigcup_{n=1}^{\infty} \operatorname{spt}\left(\phi_{n}\right)$ is closed.

Claim 4. If $\xi=\left\{x_{n}\right\}_{n=1}^{\infty}$ is in $l^{\infty}$ then

$$
f_{\xi}(s)=\sum_{n=1}^{\infty} x_{n} \phi_{n}(s)
$$

for all $s$ in $S$, defines an element $f_{\xi}$ of $C(S)$ and $\left\|f_{\xi}\right\|_{\infty}=\|\xi\|_{\infty}$.

Claim 5. The map $T:\left(l^{\infty}, \beta\right) \rightarrow C(S)_{\beta}$ defined by $T(\xi)=f_{\xi}$ for all $\xi$ in $l^{\infty}$ is a continuous linear map.

If we assume these claims for the time being (we will return to their proofs afterwards), we may complete the proof of the theorem. By Claim 5, $T$ has a well-defined adjoint map $T^{*}: M(S) \rightarrow l^{1}$, which is continuous when both range and domain have their $\beta$-weak* topologies. Thus, $T^{*}(H)$ is a $\beta$-weak* (i.e., weak) countably compact subset of $l^{1}$. By a theorem of Eberlein [8, p. 430], $T^{*}(H)$ is weakly 
conditionally compact and hence, by Theorem $2.4, \beta$-equicontinuous in $l^{1}$. Now if $\mu$ is in $M(S)$ and $\xi=\left\{x_{n}\right\}_{n=1}^{\infty}$ is in $l^{\infty}$ then

$$
\left\langle\xi, T^{*}(\mu)\right\rangle=\int f_{\xi} d \mu=\sum_{n=1}^{\infty} x_{n} \int \phi_{n} d \mu,
$$

so that

$$
T^{*}(\mu)=\left\{\int \phi_{n} d \mu\right\}_{n=1}^{\infty} .
$$

Interpreting our $\beta$-equicontinuity condition for $T^{*}(H) \subset l^{1}$, we can find an integer $N$ such that

$$
\sum_{n=N+1}^{\infty}\left|\int \phi_{n} d \mu\right|<\varepsilon / 8
$$

for all $\mu$ in $H$. In particular, if $n>N$ then $\left|\int \phi_{n} d \mu_{n}\right|<\varepsilon / 8$; but

$$
\left|\mu_{n}\left(U_{n}\right)\right| \leqq\left|\mu_{n}\right|\left(U_{n}\right)<\left|\int \phi_{n} d \mu_{n}\right|+\varepsilon / 8
$$

by Claim 1 (d). Combining these last two facts we obtain a contradiction to Claim 1 (c) whenever $n>N$. This completes the proof, modulo substantiating our five claims.

Proof of Claim 1. Let $K_{1}=D_{1}$; then there is a measure $\mu_{1}$ in $H$ such that $\left|\mu_{1}\right|\left(S \backslash K_{1}\right)>\varepsilon$. Hence, there is a compact set $C \subset S \backslash K_{1}$ such that $\left|\mu_{1}(C)\right|>\varepsilon / 4$ (combine inner regularity with a well-known result $[8$, p. 97]). Choose an open set $U_{1}$ such that $U_{1}^{-}$is compact, $C \subset U_{1} \subset U_{1}^{-} \subset S \backslash K_{1}$, and $\left|\mu_{1}\right|\left(U_{1} \mid C\right)<\left(\frac{1}{2}\right)\left[\left|\mu_{1}(C)\right|-\varepsilon / 4\right]$. Thus $\left|\mu_{1}\left(U_{1}\right)\right|>\varepsilon / 4$. Restricting the measure $\mu_{1}$ to $U_{1}$ and applying the Riesz representation theorem, we can find a function $\phi_{1} \in C_{\mathrm{c}}(S)$ such that $\left\|\phi_{1}\right\|_{\infty}=1$, spt $\left(\phi_{1}\right) \subset U_{1}$ and $\left|\mu_{1}\right|\left(U_{1}\right)<\left|\int \phi_{1} d \mu_{1}\right|+\varepsilon / 8$.

Since $K_{1} \cup U_{1}^{-}$is compact we can find a compact set $K_{2}$ with $K_{1} \cup U_{1}^{-} \subset$ int $K_{2}$. The rest of the induction follows in a similar manner.

Proof of Claim 2. This is obvious from (b) of Claim 1, since $S=\bigcup_{n=1}^{\infty} D_{n}$.

Proof of Claim 3. If $s \in F^{-}$then $s \in$ int $K_{n}$ for some $n \geqq 1$. Hence, for every open neighborhood $W$ of $s$ contained in $K_{n}$ we have that

$$
W \cap \bigcup_{i=1}^{n-1} \operatorname{spt}\left(\phi_{i}\right)=W \cap F \neq \square .
$$

Thus, $s \in \bigcup_{i=1}^{n-1} \operatorname{spt}\left(\phi_{i}\right) \subset F$ and $F$ is closed.

Proof of Claim 4. Clearly $f_{\xi}$ is well defined since at most one term in the sum is not zero. Also, for this same reason, $\left|f_{\xi}(s)\right|=\sum_{n=1}^{\infty}\left|x_{n}\right|\left|\phi_{n}(s)\right|$ for all $s \in S$; and, since each $\left|\phi_{n}\right|$ achieves its maximum of $1,\left\|f_{\xi}\right\|_{\infty}=\|\xi\|_{\infty}$. To see that $f_{\xi}$ is continuous let $s \in S$ and let $\left\{s_{i}\right\}$ be a net in $S$ such that $s_{i} \rightarrow s$. If $s \notin F$ then $F$ is closed implies there is an $i_{0}$ such that for $i \geqq i_{0} s_{i} \notin F$. Hence, for $i \geqq i_{0} f_{\xi}\left(s_{i}\right)=f_{\xi}(s)=0$ and $f_{\xi}\left(s_{i}\right) \rightarrow f_{\xi}(s)$. 
If $s \in F$ then $s \in \operatorname{spt}\left(\phi_{n}\right) \subset U_{n}$ for a unique integer $n$. Therefore there is an $i_{0}$ such that for $i \geqq i_{0} s_{i} \in U_{n}$. But then for $i \geqq i_{0}$ we have

and so $f_{\xi} \in C(S)$.

$$
f_{\xi}\left(s_{i}\right)=x_{n} \phi_{n}\left(s_{i}\right) \rightarrow x_{n} \phi_{n}(s)=f_{\xi}(s),
$$

Proof of Claim 5. Clearly $T$ is linear. Let $\left\{\xi_{i}\right\}$ be a net in $l^{\infty}$ such that $\xi_{i} \rightarrow 0(\beta)$; we must show that $T\left(\xi_{i}\right) \rightarrow 0(\beta)$ in $C(S)$. Toward this end, let $\phi \in C_{0}(S)$. If $\varepsilon>0$ then there is an integer $N$ such that for $s \notin K_{N},|\phi(s)| \leqq \varepsilon$. Thus, for $n \geqq N$, spt $\left(\phi_{n}\right) \cap K_{N}=\square$ implies $\left\|\phi \phi_{n}\right\|_{\infty} \leqq \varepsilon$. That is,

$$
\xi=\left\{\left\|\phi \phi_{n}\right\|\right\}_{n=1}^{\infty} \in c_{0} .
$$

Since $\xi_{i} \rightarrow 0(\beta)$, there is for every $\varepsilon>0$ an $i_{\varepsilon}$ such that $\left\|\xi \xi_{i}\right\|_{\infty} \leqq \varepsilon$ for $i \geqq i_{\varepsilon}$. If $\xi_{i}=\left\{x_{n}^{(i)}\right\}_{n=1}^{\infty}$ for every $i$ then for $i \geqq i_{\varepsilon}$ we have

$$
\begin{aligned}
\left\|\phi T\left(\xi_{i}\right)\right\|_{\infty} & =\sup \left\{\sum_{n=1}^{\infty}\left|x_{n}^{(i)}\right|\left|\phi(s) \phi_{n}(s)\right|: s \in S\right\} \\
& =\sup \left\{\left|x_{n}^{(i)}\right|\left|\phi(s) \phi_{n}(s)\right|: s \in S, n \geqq 1\right\} \\
& =\sup \left\{\left|x_{n}^{(i)}\right|\left\|\phi \phi_{n}\right\|_{\infty}: n \geqq 1\right\} \leqq \varepsilon .
\end{aligned}
$$

Thus, $T\left(\xi_{i}\right) \rightarrow 0(\beta)$ and $T$ is continuous.

This concludes the proof of the theorem.

Note that the reason for condition (b) on the sequence of quadruples was to ensure that $\bigcup_{n=1}^{\infty} K_{n}$ was both open and closed. From this it followed that $F$ was closed and $f_{\xi}$ was continuous for every $\xi$ in $l^{\infty}$. This same method yields a proof if $S$ is a locally compact topological group, and (b) is replaced by the requirement that each $K_{n}$ be a compact symmetric neighborhood of the identity and $K_{n}^{2} \cup U_{n}^{-} \subset$ int $K_{n+1}$. Then $\bigcup_{n=1}^{\infty} K_{n}$ is an open subgroup of $S$ and therefore also closed. Both of these proofs can be subsumed under the proof of the case where $S$ is paracompact. By a standard theorem on paracompactness [1, p. 107], $S$ can be expressed as the union of a collection $\left\{S_{a}: a \in A\right\}$ of pairwise disjoint open and closed $\sigma$-compact subsets of $S$. Let $S_{a}=\bigcup_{n=1}^{\infty} C(a, n)$, where each $C(a, n)$ is compact and $C(a, n) \subset$ int $C(a, n+1)$ for $n \geqq 1$ and $a \in \mathscr{A}$. By an induction argument similar to that used in the above proof, we obtain a strictly increasing sequence of integers $\left\{k_{n}\right\}$ and a sequence $\left\{a_{n}\right\}$ in $\mathscr{A}$, as well as the sequence $\left\{\left(\mu_{n}, \phi_{n}, K_{n}, U_{n}\right)\right\}$. This sequence of quadruples has all the properties it had in the proof of the theorem except that condition (b) is replaced by

$$
\text { (b') } K_{n}=\bigcup_{i=1}^{k_{n}} C\left(k_{n}, a_{i}\right) \text { and } U_{n}^{-} \subset \text { int } K_{n+1} \text {. }
$$

We now proceed as above and

$$
\begin{aligned}
\bigcup_{n=1}^{\infty} K_{n} & =\bigcup_{n=1}^{\infty} \bigcup_{i=1}^{k_{n}} C\left(k_{n}, a_{i}\right) \\
& =\bigcup_{n=1}^{\infty} S_{a_{n}}
\end{aligned}
$$

since $k_{n+1}>k_{n}$. Thus $\bigcup_{n=1}^{\infty} K_{n}$ is both open and closed. 
REMARKS. There is a class of spaces for which the preceding method of proof cannot succeed-namely, the pseudocompact, noncompact spaces. In such a space, no matter how the sets $K_{n}$ are chosen $F$ will never be closed [9].

There are spaces for which Theorem 2.6 does not hold, as the following example (of a pseudocompact space) illustrates. Let $\Omega_{0}$ be the space of ordinal numbers less than the first uncountable ordinal with the order topology. We will show that $C\left(\Omega_{0}\right)_{\beta}$ is not a Mackey space.

Let $H$ be the $\beta$-weak* closed convex circled hull of the set of all measures of the form

$$
\frac{1}{2}\left[\delta_{s}-\delta_{s+1}\right]
$$

where $s$ is a nonlimit ordinal and $s+1$ is its immediate successor. We will need the following facts: $\Omega_{0}$ is not $\sigma$-compact; every continuous function $f$ on $\Omega_{0}$ is eventually constant (i.e., there is an $x$ in $\Omega_{0}$ such that $f(y)=f(x)$ whenever $y \geqq x$ ), and hence, the Stone-Čech and one-point compactifications of $\Omega_{0}$ are the same [12]. Also, if $s \in \Omega_{0}$ then the characteristic function of $[1, s]$ is continuous.

Let $\Omega$ be the first uncountable ordinal and $\Omega_{1}$ the Stone-Čech compactification of $\Omega_{0}$. Hence $\Omega_{1}=\Omega_{0} \cup\{\Omega\}$ and $M\left(\Omega_{1}\right)=M\left(\Omega_{0}\right) \oplus \mathbf{C}\left\{\delta_{\Omega}\right\}$, where

$$
\mathbf{C}\left\{\delta_{\Omega}\right\}=\left\{c \delta_{\Omega}: c \in \mathbf{C}\right\} .
$$

We claim that if we consider $H$ as a subset of $M\left(\Omega_{1}\right)$ then $H$ is weak* closed. If this is so then we would have $H$ weak* compact in $M\left(\Omega_{1}\right)$ since $H$ is clearly contained in the unit ball [8, p. 424]. But the weak* topology of $M\left(\Omega_{1}\right)$ relativized to $M\left(\Omega_{0}\right)$ is the $\beta$-weak* topology and this would imply that $H$ is $\beta$-weak* compact. Since, by Theorem $2.2, H$ is not $\beta$-equicontinuous, we would have that $C\left(\Omega_{0}\right)_{\beta}$ is not a Mackey space.

To prove the above claim, suppose that $\mu$ is in the weak* closure of $H$ in $M\left(\Omega_{1}\right)$. Then there is a unique measure $\nu$ in $M\left(\Omega_{0}\right)$ and a scalar $c$ such that $\mu=\nu+c \delta_{\Omega}$. We must show that $c=0$. Clearly $\lambda\left(\Omega_{1}\right)=0$ for each $\lambda$ in $H$, and so $\mu\left(\Omega_{1}\right)=0$. Thus, $c=-\nu\left(\Omega_{1}\right)=-\nu\left(\Omega_{0}\right)$. Since $\Omega_{0}$ is not $\sigma$-compact and $\nu$ vanishes off a $\sigma$-compact set, there is a limit ordinal $x<\Omega$ such that $\nu$ vanishes off $[1, x]$. If $s$ is any nonlimit ordinal then either $s<x$ or $x<s$. If $s<x$ then' $s+1<x$; so if $f$ is the characteristic function of $[1, x], f(s)=f(s+1)=1$. If $x<s$ then $f(s)=f(s+1)=0$. In either case $\int f d \lambda=0$ for all $\lambda$ in $H$ and this implies

$$
0=\int f d \mu=\int f d \nu+c f(\Omega)=\int f d \nu=\nu([1, x])=\nu\left(\Omega_{0}\right) .
$$

Thus $c=0$ and $\mu=\nu \in M\left(\Omega_{0}\right)$. But the weak* topology of $M\left(\Omega_{1}\right)$ relativized to $M\left(\Omega_{0}\right)$ is the $\beta$-weak* topology and $H$ is $\beta$-weak* closed. Therefore $\mu \in H$ and $C\left(\Omega_{0}\right)_{\beta}$ is not a Mackey space.

3. Subspaces of $C(S)_{\beta}$, the space $\left(l^{\infty}, \beta\right)$, and $\left(H^{\infty}, \beta\right)$. A natural question to ask is whether or not a subspace of $C(S)_{\beta}$ is a Mackey space provided $C(S)_{\beta}$ is. Along these lines it is known that the completion of a Mackey space is a Mackey space but the converse is false. In fact, $C(S)_{\beta}$ is the completion of $C_{0}(S)_{\beta}$, but if $S$ is not 
compact then $C_{0}(S)_{\beta}$ is not a Mackey space since the norm topology yields $M(S)$ as the adjoint of $C_{0}(S)$ and is properly stronger than $\beta$.

The difficulties in attacking the general problem may be visualized as follows. Let $E$ be a $\beta$-closed subspace of $C(S)$ and let $i: E_{\beta} \rightarrow C(S)_{\beta}$ be the injection mapping with $i^{*}: M(S) \rightarrow E_{\beta}^{*}$ its adjoint map. In order to show that a subset $H \subset E_{\beta}^{*}$ is $\beta$-equicontinuous it is necessary and sufficient to find a $\beta$-equicontinuous set $H_{1} \subset M(S)$ such that $i^{*} H_{1}=H$. Therefore if $C(S)_{\beta}$ is a Mackey space and $H \subset E_{\beta}^{*}$ is a $\beta$-weak* compact convex circled set then to show that $H$ is $\beta$-equicontinuous we must find a $\beta$-weak* compact convex circled subset $H_{1} \subset M(S)$ such that $i^{*} H_{1}=H$. Since $E_{\beta}^{*}$ with its $\beta$-weak* topology is topologically isomorphic to some quotient space of $M(S)$ and $i^{*}$ acts like the canonical map, it would seem that what is needed is a version of a theorem of Bartle and Graves (e.g., see p. 375 of [14]), where both domain and range have their $\beta$-weak* topologies. No such result is presently available, although in the case of $l^{\infty}$ this theorem can be used to great advantage (see Theorem 3.2 below).

Recall that we used a theorem of J. Schür to prove that $\left(l^{\infty}, \beta\right)$ is a strong Mackey space. It is not difficult to prove Schür's result if we assume that $\left(l^{\infty}, \beta\right)$ is a strong Mackey space. A statement similar to Schür's theorem will be what is needed to characterize the $\beta$-closed subspaces of $l^{\infty}$ which are strong Mackey spaces.

Lemma 3.1. Let $E$ be a $\beta$-closed subspace of $l^{\infty}$. Then $E_{\beta}$ is semireflexive and $E_{\beta}^{*}$ with its strong topology is a Banach space. Consequently, the $\beta$-weak* topology on $E_{B}^{*}$ is exactly its weak topology which it has as a Banach space.

Proof. Since $\left(l^{\infty}, \beta\right)$ is semireflexive (Lemma 2.3) $E_{\beta}$ is semireflexive. Also, $E_{B}^{*}$ is topologically isomorphic to $l^{1} / N$ with its quotient norm, where $N=E^{\perp} \subset l^{1}$ $\left[13\right.$, p. 190]. Hence $E_{B}^{*}$ is a Banach space.

THEOREM 3.2. If $E$ is a closed subspace of $\left(l^{\infty}, \beta\right)$ then a subset of $E_{\beta}^{*}$ is $\beta$-equicontinuous if and only if it is norm conditionally compact.

Proof. Let $H \subset E_{\beta}^{*}$ be $\beta$-equicontinuous and let $i: E_{\beta} \rightarrow\left(l^{\infty}, \beta\right)$ be the injection map. Then there is a $\phi \in c_{0}$ such that $i^{*}\left(V_{\phi}^{0}\right) \supset H$, where $i^{*}: l^{1} \rightarrow E_{\beta}^{*}$ is the adjoint map of $i$. But $V_{\phi}^{0}$ is norm compact in $l^{1}$ by Theorem 2.4 and, since $i^{*}$ is norm continuous, we have that $H$ has norm compact closure in $E_{\beta}^{*}$.

Assume that $H$ is norm compact. Then $E_{\beta}^{*}$ is a Banach space and $i^{*}$ is a bounded linear map of $l^{1}$ onto $E_{\beta}^{*}$. By the Bartle-Graves selection theorem [14, p. 375], there exists a continuous function $f: E_{\beta}^{*} \rightarrow l^{1}$ such that $f(I) \in i^{*-1}(I)$ for all $I$ in $E_{B}^{*}$. Therefore $f(H)$ is norm compact in $l^{1}$ and $i^{*}(f(H))=H$ imply that $H$ is $\beta$-equicontinuous.

THEOREM 3.3. If $E$ is a $\beta$-closed subspace of $l^{\infty}$ then the following are equivalent:

(a) $E_{\beta}$ is a Mackey space;

(b) $E_{\beta}$ is a strong Mackey space;

(c) every $\beta$-weak ${ }^{*}$ compact set in $E_{\beta}^{*}$ is norm compact;

(d) every $\beta$-weak* convergent sequence in $E_{\beta}^{*}$ is norm convergent. 
Proof. (a) $\Leftrightarrow(\mathrm{b})$. Since the $\beta$-weak* and weak topologies on $E_{\beta}^{*}$ are the same (Lemma 3.1), the $\beta$-weak* closed convex circled hull of a $\beta$-weak* compact subset of $E_{\beta}^{*}$ is $\beta$-weak* compact [8, p. 434].

(b) $\Rightarrow$ (c). If $H \subset E_{\beta}^{*}$ is $\beta$-weak* compact then $H$ is $\beta$-equicontinuous and hence norm compact by Theorem 3.2.

(d) $\Rightarrow$ (b). If $H$ is $\beta$-weak* compact then by Lemma 3.1 and $[8$, p. 430] it is $\beta$ weak* sequentially compact. It now follows from (d) that $H$ is norm compact and, hence, $\beta$-equicontinuous.

To complete the proof of the theorem we need only show that (c) $\Rightarrow(d)$, and this is a triviality.

Now let us turn our attention to $H^{\infty}$, the space of bounded holomorphic functions on the open unit disk $D$. Theorems 3.4 and 3.5 below were obtained by Rubel and Shields [15]. We present them here because they form a direct path to our result that $\left(H^{\infty}, \beta\right)$ is not a Mackey space.

We follow a method of Brown, Shields, and Zeller [2] and find a sequence $\left\{a_{n}\right\}$ in $D$ having no limit points in $D$, and such that

$$
\|f\|_{\infty}=\sup \left\{\left|f\left(a_{n}\right)\right|: n \geqq 1\right\}
$$

for all $f$ in $H^{\infty}$. Hence, if $E$ is the subspace of $l^{\infty}$ consisting of all sequences $\left\{f\left(a_{n}\right)\right\}_{n=1}^{\infty}$ where $f$ is in $H^{\infty}$ then $T: H^{\infty} \rightarrow E$ defined by

$$
T(f)=\left\{f\left(a_{n}\right)\right\}_{n=1}^{\infty}
$$

for $f$ in $H^{\infty}$, is an isometry. Moreover, since $\left\{a_{n}\right\}$ has no limit points in $D$ it is a discrete sequence. Thus, if $\left\{f_{i}\right\}$ is a net in $H^{\infty}$ such that $f_{i} \rightarrow 0(\beta)$ and $\xi=\left\{x_{n}\right\}_{n=1}^{\infty}$ is a sequence in $c_{0}$, then there is a function $\phi$ in $C_{0}(D)$ such that $\phi\left(a_{n}\right)=x_{n}$ for all $n \geqq 1$ [5, p. 96]. If $\varepsilon>0$ then there is an $i_{0}$ such that for $i \geqq i_{0}\left\|f_{i} \phi\right\|_{\infty} \leqq \varepsilon$. But this implies that for $i \geqq i_{0}$

$$
\begin{aligned}
\left\|T\left(f_{i}\right) \xi\right\|_{\infty} & =\sup \left\{\left|f_{i}\left(a_{n}\right) x_{n}\right|: n \geqq 1\right\} \\
& =\sup \left\{\left|f_{i}\left(a_{n}\right) \phi\left(a_{n}\right)\right|: n \geqq 1\right\} \\
& \leqq\left\|f_{i} \phi\right\|_{\infty} \leqq \varepsilon
\end{aligned}
$$

and so $T\left(f_{i}\right) \rightarrow 0(\beta)$ in $E$. Therefore

$$
T:\left(H^{\infty}, \beta\right) \rightarrow E_{\beta}
$$

is continuous. Not only will we use this fact later but the continuity of $T$ can be used to give an alternate proof of the following results of Rubel and Shields (see p. 80 of [7]).

THEOREM 3.4. A subset of $\left(H^{\infty}, \beta\right)$ is compact if and only if it is closed and bounded.

THEOREM 3.5. If I is a linear functional on $H^{\infty}$ which is $\beta$-continuous on the unit ball of $H^{\infty}$ then I is $\beta$-continuous on $H^{\infty}$. Hence, $\left(H^{\infty}, \beta\right)^{*}$ with its strong topology is a Banach space and $H^{\infty}$ is its adjoint. 
Corollary 3.6. A linear functional I on $H^{\infty}$ is $\beta$-continuous if and only if there is a Lebesgue integrable function $g$ on $[-\pi, \pi]$ such that

or all $f$ in $H^{\infty}$.

$$
I(f)=\frac{1}{2 \pi} \int_{-\pi}^{\pi} f\left(e^{i \theta}\right) g(\theta) d \theta
$$

Proof. Let $g$ be a Lebesgue integrable function on $[-\pi, \pi]$ and let $I$ be defined as above. If $B$ is the closed unit ball in $H^{\infty}$ then the $\beta$ and c-op topologies agree on $B$ and so $(B, \beta)$ is metrizable. If $\left\{f_{n}\right\}$ is a sequence in $B$ such that $f_{n} \rightarrow f$ in $(B, \beta)$ then identify each $f_{n}$ and $f$ with its boundary values on the unit circle. Hence $\left\{f_{n}\right\}$ is a sequence in the unit ball of $L^{\infty}=L^{\infty}(-\pi, \pi)$, the adjoint of the separable Banach space $L^{1}=L^{1}(-\pi, \pi)$. Hence, there is a subsequence $\left\{f_{n_{k}}\right\}$ of $\left\{f_{n}\right\}$ and an element $h$ in the unit ball of $L^{\infty}$ such that $f_{n_{k}} \rightarrow h \sigma\left(L^{\infty}, L^{1}\right)$ (i.e., the topology induced on $L^{\infty}$ by $L^{1}$ ). This implies that

$$
\hat{h}(n)=\frac{1}{2 \pi} \int_{-\pi}^{\pi} h(\theta) e^{-i n \theta} d \theta=\lim _{k} \hat{f}_{n_{k}}(n)
$$

for $n=0, \pm 1, \pm 2, \ldots$ (here, $\hat{h}$ is the Fourier-Stieltjes transform of $h$ [16]). Hence $\hat{h}(n)=0$ for $n<0$ and for $n \geqq 0 \hat{f}_{n_{k}}(n)=(1 / n !) f_{n_{k}}^{(n)}(0)$. But $f_{n_{k}} \rightarrow f(\beta)$ in $H^{\infty}$ implies

$$
\begin{aligned}
\hat{f}(n) & =(1 / n !) f^{(n)}(0)=\lim _{k}(1 / n !) f_{n_{k}}^{(n)}(0) \\
& =\lim _{k} \hat{f}_{n_{k}}(n) .
\end{aligned}
$$

Therefore $\hat{h}(n)=\hat{f}(n)$ for all $n$ and so $h(\theta)=f\left(e^{i \theta}\right)$ for almost all $\theta$. What we have shown is that every $\sigma\left(L^{\infty}, L^{1}\right)$ convergent subsequence of $\left\{f_{n}\right\}$ converges to $f$. Since the unit ball of $L^{\infty}$ is $\sigma\left(L^{\infty}, L^{1}\right)$ compact, this implies that $f_{n} \rightarrow f \sigma\left(L^{\infty}, L^{1}\right)$. Therefore $I\left(f_{n}\right) \rightarrow I(f)$ and $I$ is continuous on $(B, \beta)$. By the above theorem we have that $I$ is in $\left(H^{\infty}, \beta\right)^{*}$.

For the converse let $u: H^{\infty} \rightarrow L^{\infty}$ be the imbedding which takes each function in $H^{\infty}$ into the class in $L^{\infty}$ represented by its boundary values. The first part of this proof established that $u$ is continuous if $H^{\infty}$ has its weak topology from $\left(H^{\infty}, \beta\right)^{*}$ and $L^{\infty}$ its $\sigma\left(L^{\infty}, L^{1}\right)$ topology. If $E=u\left(H^{\infty}\right)$ and $I$ is in $\left(H^{\infty}, \beta\right)^{*}$ then, by an argument similar to that used in the alternate proof of the preceding theorem of Rubel and Shields (see Theorem 5 of [7]), we have that $I \circ u^{-1}$ is a $\sigma\left(L^{\infty}, L^{1}\right)$ continuous linear functional on $E$. As such there is a Lebesgue integrable function $g$ on $[-\pi, \pi]$ such that

$$
I \circ u^{-1}(h)=\frac{1}{2 \pi} \int_{-\pi}^{\pi} h(\theta) g(\theta) d \theta
$$

for all $h$ in $E$. Therefore if $f$ is in $H^{\infty}$ then

$$
I(f)=I \circ u^{-1}(u(f))=\frac{1}{2 \pi} \int_{-\pi}^{\pi} f\left(e^{i \theta}\right) g(\theta) d \theta .
$$

TheOREM 3.7. A subset of $\left(H^{\infty}, \beta\right)^{*}$ is $\beta$-equicontinuous if and only if it is norm conditionally compact. 
Proof. Recall that by Theorem $3.5\left(H^{\infty}, \beta\right)^{*}$ is a Banach space where the norm of an element $I$ is given by

$$
\|I\|=\sup \left\{|I(f)|: f \in H^{\infty},\|f\|_{\infty} \leqq 1\right\} .
$$

If $A \subset\left(H^{\infty}, \beta\right)^{*}$ is norm compact and $T:\left(H^{\infty}, \beta\right) \rightarrow E_{\beta}$ is the map described prior to Theorem 3.4, then $T^{*}: E_{\beta}^{*} \rightarrow\left(H^{\infty}, \beta\right)^{*}$ is an isometry onto $\left(H^{\infty}, \beta\right)^{*}$. Therefore $T^{*-1}(A)$ is norm compact in $E_{\beta}^{*}$ and hence, by Theorem $3.2, T^{*-1}(A)$ is $\beta$ equicontinuous. This implies there is a $\phi \in c_{0}$ such that $T^{*-1}(A) \subset V_{\phi}^{0}$. But $T$ is continuous and so $T^{-1}\left(V_{\phi}\right)$ is a $\beta$-neighborhood of zero in $H^{\infty}$. It is routine to show that $A \subset\left[T^{-1}\left(V_{\phi}\right)\right]^{0}$ and hence $A$ is $\beta$-equicontinuous.

Now suppose that $A \subset\left(H^{\infty}, \beta\right)^{*}$ is $\beta$-equicontinuous and $\left\{I_{n}\right\}$ is a sequence in $A$. Then $A$ is $\beta$-weak* conditionally compact; since the $\beta$-weak* topology on $\left(H^{\infty}, \beta\right)^{*}$ is the same as its weak topology which it has as a Banach space (Theorem 3.5), the Eberlein-Smulyan theorem [8, p. 430] implies that $A$ is $\beta$-weak* sequentially compact. Therefore some subsequence of $\left\{I_{n}\right\}$ converges $\beta$-weak* to an element $I$ of $\left(H^{\infty}, \beta\right)^{*}$. Let us suppose, for notational reasons, that $I_{n} \rightarrow I \beta$-weak*. Since the unit ball of $H^{\infty}$ is $\beta$-compact and circled, there is for each integer $n$ a function $f_{n}$ in ball $\left(H^{\infty}\right)$ with

$$
\left\|I-I_{n}\right\|=\left(I-I_{n}\right)\left(f_{n}\right) .
$$

Also, since ball $\left(H^{\infty}\right)$ with the $\beta$ topology is a compact metric space, there is a subsequence $\left\{f_{n_{k}}\right\}$ of $\left\{f_{n}\right\}$ and an element $f$ of ball $\left(H^{\infty}\right)$ such that $f_{n_{k}} \rightarrow f(\beta)$. Since $\left\{I-I_{n}\right\}$ is $\beta$-equicontinuous, for any $\varepsilon>0$ there is an integer $N$ such that for $n_{k} \geqq N$

$$
\left|\left(I-I_{n}\right)\left(f-f_{n_{k}}\right)\right|<\varepsilon / 2 \text { for all } n \geqq 1 \text {; and also, }\left|I_{n_{k}}(f)-I(f)\right|<\varepsilon / 2 .
$$

It now follows that for $n_{k} \geqq N$

$$
\left\|I-I_{n_{k}}\right\|<\varepsilon
$$

and the theorem is proved.

CoRollary 3.8. If $\left\{I_{n}\right\}, I$ are in $\left(H^{\infty}, \beta\right)^{*}$ then $I_{n} \rightarrow I$ in norm if and only if $I_{n} \rightarrow I \beta$-weak ${ }^{*}$ and $\left\{I_{n}\right\}$ is $\beta$-equicontinuous.

THEOREM 3.9. $\left(H^{\infty}, \beta\right)$ is not a Mackey space.

Proof. Since $\left(H^{\infty}, \beta\right)^{*}$ with its strong topology is a Banach space with $H^{\infty}$ as adjoint, it is sufficient to show that $\left(H^{\infty}, \beta\right)$ is not a strong Mackey space $[9$, p. 434]. For each integer $n \geqq 1$ define

$$
I_{n}(f)=\frac{1}{2 \pi} \int_{-\pi}^{\pi} f\left(e^{i \theta}\right) e^{-i n \theta} d \theta
$$

for all $f$ in $H^{\infty}$. Then $I_{n} \in\left(H^{\infty}, \beta\right)^{*}$ by Corollary 3.6. Also $I_{n}(f)=\hat{f}(n)$, the FourierStieltjes transform of the function given by the boundary values of $f$. Therefore $I_{n} \rightarrow 0 \beta$-weak* (see [16]). Clearly $\left\|I_{n}\right\| \leqq 1$ and since $I_{n}(f)=1$ when $f(z)=z^{n}$, we have $\left\|I_{n}\right\|=1$ for all $n$. Therefore $\left\{I_{n}\right\}$ cannot converge to zero in norm and, by the preceding corollary, $\left\{I_{n}\right\}$ is not $\beta$-equicontinuous. This concludes the proof. 
REMARK. Theorem 3.9 answers a question posed by Rubel and Shields.

If $N$ is a closed subspace of $l^{1}$ such that there is a bounded projection onto $N$ then $E_{\beta}$ is a Mackey space, where $E=N^{\perp} \subset l^{\infty}$. If $E$ is the image of $H^{\infty}$ under the map $T$ described prior to Theorem 3.4, then it is easy to see that $E_{\beta}$ is not a Mackey space. Hence, if $N=E^{\perp} \subset l^{1}$ then there is no bounded projection of $l^{1}$ onto $N$. Also, this space $E$ is an example of a closed subspace of a semireflexive Mackey space $\left(l^{\infty}, \beta\right)$ which is not a Mackey space.

\section{BIBLIOGRAPHY}

1. N. Bourbaki, Éléments de mathématique, Livre III, Topologie général, Actualités Sci. Ind. No. 1142, Hermann, Paris, 1951.

2. L. Brown, A. Shields and K. Zeller, On absolutely convergent exponential sums, Trans. Amer. Math. Soc. 96 (1960), 162-183.

3. R. C. Buck, Operator algebras and dual spaces, Proc. Amer. Math. Soc. 3 (1952), 681-687.

4. - Algebraic properties of classes of analytic functions, Seminars on analytic functions. II, pp. 175-188, Institute for Advanced Study, Princeton, N. J., 1957.

5. - Bounded continuous functions on a locally compact space, Michigan Math. J. 5 (1958), 95-104.

6. J. B. Conway, The strict topology and compactness in the space of measures, Bull. Amer. Math. Soc. 72 (1966), 75-78.

7. - Subspaces of $C(S)_{\beta}$, the space $\left(l^{\infty}, \beta\right)$, and $\left(H^{\infty}, \beta\right)$, Bull. Amer. Math. Soc. 72 (1966), 79-81.

8. N. Dunford and J. Schwartz, Linear operators, Part I, Interscience, New York, 1958.

9. I. Glicksberg, The representation of functionals by integrals, Duke Math. J. 19 (1952), 253-261.

10. - Bishop's generalized Stone-Weierstrass theorem for the strict topology, Proc. Amer. Math. Soc. 14 (1963), 329-333.

11. C. S. Herz, The spectral theory of bounded functions, Trans. Amer. Math. Soc. 94 (1960), 181-232.

12. J. L. Kelley, General topology, Van Nostrand, New York, 1955.

13. J. L. Kelley, I. Namioka, et al, Linear topological spaces, Van Nostrand, Princeton, N. J., 1963.

14. E. Michael, Continuous selections. I, Ann. of Math. 63 (1956), 361-382.

15. L. Rubel and A. Shields, Weak topologies on the bounded holomorphic functions, Bull. Amer. Math. Soc. 71 (1965), 349-352.

16. W. Rudin, Fourier analysis on groups, Interscience, New York, 1962.

17. C. Todd, Stone-Weierstrass theorems for the strict topology, Proc. Amer. Math. Soc. 16 (1965), 654-659.

18. Ju-kwei Wang, Multipliers of commutative Banach algebras, Pacific J. Math. 11 (1961), 1131-1149.

19. J. Wells, Multipliers of ideals in function algebras, Duke Math. J. 31 (1964), 703-709.

20. - Bounded continuous vector valued functions on a locally compact space, Michigan

Math. J. 12 (1965), 119-126.

Louisiana State University, Baton Rouge, Louisiana

INDIANA UNIVERSITY, BLOOMINGTON, INDIANA 